\title{
COLEDOCOLITIASIS: \\ DIAGNÓSTICO Y MANEJO
}

\author{
Guillermo Aldana Dimas MD*, Ricardo Mora Posada MD**, Carlos Alberto Millán MD**
}

\section{Resumen}

La coledocolitiasis puede ocurrir hasta en el $15 \%$ de los casos de colelitiasis. En la era moderna de la colecistectomía laparoscópica se ha planteado un debate sobre el manejo de la coledocolitiasis previo a la realización del procedimiento quirúrgico. Varios estudios han investigado el uso de criterios preoperatorios, como las pruebas de función hepática, los hallazgos ecográficos y la clínica del paciente, para predecir el riesgo de cálculos en el colédoco. Los resultados varían un poco entre los estudios y de ellos se han desprendido diferentes esquemas y algoritmos para orientar el manejo, dentro del cual se incluyen técnicas tradicionales de cirugía abierta y las endoscópicas y laparoscópicas de más reciente aparición.

Palabras clave: coledocolitiasis, fosfatasa alcalina, bilirruìinas, alaninoaminotransferasa (TGP-ALT), aspartatoaminotransferasa (TGO-AST).

\section{DIAGNOSIS AND MANAGEMENT OF CHOLEDOCHOLITHIASIS}

\begin{abstract}
Choledocholithiasis may occur in up to $15 \%$ of cases of cholelithiasis. A debate has been posed in the modern era of laparoscopic cholecystectomy on how to handle choledocholithiasis before conducting the surgical procedure. Several studies have analyzed the use of preoperative criteria, such as liver function tests, ultrasound and clinical findings of the patient, in order to predict the presence of gallstones in the common bile duct. Results vary between studies and different regimes and algorithms to guide management have derived from them, including traditional open surgery techniques and most recent endoscopic and laparoscopic techniques.
\end{abstract}

Key words: choledocholithiasis, alkaline phosphatase, bilirrubin, alanine aminotransferase (SGPT-ALT), aspartate aminotransferase (SGOT-AST).

Fecha recibido: abril 12 de 2011 - Fecha aceptado: mayo 18 de 2011

* Jefe del Servicio de Cirugía General, Hospital de San José. Profesor Asistente, Fundación Universitaria de Ciencias de la Salud. Bogotá DC. Colombia.
** Residente IV año de Cirugía General, Fundación Universitaria de Ciencias de la Salud. Bogotá DC. Colombia. 


\section{Introducción}

La patología biliar es una de las enfermedades más comunes del sistema digestivo, su frecuencia es muy variable en los diferentes grupos raciales pero según informes de necropsias se estima una prevalencia de cálculos biliares entre 11 y $36 \%$ de la población, más alta en mujeres (1.22: 1), con una media de edad de presentación de 56 años. ${ }^{1}$ Entre 1 y $2 \%$ de los que exhiben colelitiasis sufren síntomas o complicaciones cada año e incluyen colecistitis aguda y crónica, colangitis, pancreatitis aguda, carcinoma de la vesícula biliar, íleo biliar y coledocolitiasis, definida como la presencia de cálculos en la vía biliar. ${ }^{2}$

Desde el punto de vista histórico la primera descripción de cálculos en el colédoco es probable que se deba al anatomista de Padua, Realdo Colombo (1516-1559), quien al efectuar la autopsia a San Ignacio de Loyola encontró cálculos en la vesícula y el colédoco y uno de estos últimos erosionó la vena porta, cuadro que es muy posible que lo llevó a la muerte por una sepsis de origen biliar. ${ }^{3}$ El cirujano neoyorquino Robert Abbé describió en 1889 la primera exploración abierta del colédoco. Otras fuentes otorgan el crédito al cirujano inglés Knowesly Thornton. De todas maneras cinco años más tarde Ludwing Rehn efectuó por primera vez el procedimiento combinado de colecistectomía y exploración de vías biliares. ${ }^{4}$

En la actualidad, ante el auge de la cirugía laparoscópica para el manejo de la patología biliar, el enfoque de los pacientes con coledocolitiasis ha requerido la utilización de diferentes parámetros clínicos, bioquímicos e imagenológicos que permitan predecir el riesgo de cálculos en el colédoco y definir la terapia.

\section{Anatomía}

La vesícula biliar es un saco piriforme situado en la fosa cística, en la cara inferior y posterior del lóbulo derecho del hígado. ${ }^{5}$ Está separada del parénquima hepático por la lámina cística que se continúa con la placa hiliar. En ocasiones se introduce en la profundidad del hígado o puede tener un mesenterio. ${ }^{6}$ Tiene una capacidad de 30 a $50 \mathrm{ml}$ y desde el punto de vista anatomopatológico se identifica fondo, cuerpo y el cuello en forma de $\mathrm{S}$, que en el lado derecho puede haber un receso a veces producido por una dilatación crónica que se proyecta hacia el duodeno, conocida como bolsa de Hartmann. ${ }^{6}$ Termina en el conducto cístico que tiene $3 \mathrm{~mm}$ de diámetro y de 2 a $4 \mathrm{~cm}$ de longitud y al unirse al conducto hepático común configuran el colédoco. Esta unión puede variar de acuerdo con su disposición angular, paralela o espiral. El conducto colédoco presenta cuatro porciones: supra y retroduodenal, pancreática e intramural, terminando en la papila duodenal mayor en una relación variable con el conducto pancreático.?

Los conductos biliares extrahepáticos reciben su perfusión sanguínea de distintas arterias importantes. Northover y Terblanche efectuaron un estudio con un molde en resina de cadáveres humanos y describieron dos grandes vasos axiales que seguían los bordes laterales del colédoco supraduodenal, en el horario de las tres y las nueve, así como una media de ocho pequeñas arterias con un diámetro de $0.3 \mathrm{~mm}$ que se originan por debajo de las arterias pancreatoduodenal superior y gastroduodenal, y en la parte superior de las arterias hepática derecha y cística. ${ }^{8}$

Los conductos biliares extra e intrahepáticos están rodeados por un fino plexo venoso que drena en las venas marginales, ${ }^{9}$ que se encuentran en las posiciones tres y nueve como los vasos arteriales. En la parte inferior drenan al plexo venoso pancreatoduodenal, y en la superior entran al hígado o se unen al plexo venoso hiliar, que acaba desembocando en ramas de la vena porta. ${ }^{10} \mathrm{El}$ plexo de los conductos biliares intrahepáticos drena en la vena porta adyacente. Las venas de la vesícula biliar no siguen a las ramas arteriales y drenan en forma directa en el hígado. ${ }^{11}$

\section{Fisiopatología}

Los cálculos de la vía biliar se clasifican de acuerdo con su origen en primarios formados en el conducto biliar, secundarios que provienen de la vesícula biliar y terciarios a partir de cálculos intrahepáticos. ${ }^{12}$ Los 
primarios son casi siempre pigmentarios marrones compuestos en variadas proporciones de sales de calcio, bilirrubina, colesterol y proteínas. Se forman por infección crónica de la bilis por bacterias entéricas (E. coli y Bacterioides), favorecido por estasis biliar que permite el depósito de mucina en los conductos biliares. Los iones hidrógeno de la bilis son neutralizados por la mucina creando un ambiente menos ácido donde el carbonato de calcio, bilirrubinas y fosfato se pueden precipitar. ${ }^{13}$

Los secundarios se componen de manera principal por colesterol y constituyen el $80 \%$ de todos los cálculos. Contienen cristales de monohidrato de colesterol y sales de calcio, además de pigmentos biliares, proteínas y ácidos grasos. En su patogenia influyen la supersaturación de colesterol o el balance irregular entre colesterol, fosfolípidos y ácidos biliares, la formación del núcleo o cuando las micelas se precipitan y forman cristales de colesterol y la hipomotilidad vesicular. ${ }^{13}$

\section{Diagnóstico}

La coledocolitiasis se sospecha en el preoperatorio ante todo paciente con ictericia, elevación de las enzimas hepáticas, la presencia de pancreatitis o colangitis, signos radiológicos de dilatación de la vía biliar o la presencia de cálculos en el colédoco. ${ }^{14}$

Las pruebas bioquímicas hepáticas muestran elevación de las bilirrubinas con énfasis en la fracción directa, transaminasas en especial la alanino amino transferasa (ALT) y la fosfatasa alcalina, las cuales pueden llegar a tener un valor predictivo negativo de más del $97 \%$ y uno positivo ante cualquier anormalidad de solo el $15 \% .{ }^{15}$ Sin embargo, algunos estudios han informado un aumento en el valor predictivo positivo ante la alteración de las bilirrubinas, la fosfatasa alcalina o la gamma glutil transpeptidasa, que puede llegar a ser del 25 al $50 \% .^{16}$

Se asume que la alteración en las pruebas hepáticas aumenta a medida que progresa la duración y la severidad de la obstrucción del conducto biliar. Es así como en el estudio realizado por Onken y colaboradores se estableció que el valor de bilirrubina total por encima de $1.7 \mathrm{mg} / \mathrm{dl}$ tenía una especificidad del 60\% para coledocolitiasis y llegaba a ser del $75 \%$ cuando el punto de corte se tomaba en $4 \mathrm{mg} / \mathrm{dl}$. Sin embargo, la media de los niveles de bilirrubina es de 1.5 a $1.9 \mathrm{mg} / \mathrm{dl}$ y solo una minoría presentó niveles por encima de $4 \mathrm{mg} / \mathrm{dl} .{ }^{17}$

El estudio de la imagen de la vía biliar se fundamenta en la ecografía transabdominal como prueba para el tamizaje de los cálculos del colédoco, pero tiene una sensibilidad baja, cerca del $30 \% .{ }^{18}$ Sin embargo, detecta de manera más fiable la dilatación de la vía biliar, colédoco mayor de $7 \mathrm{~mm}$, como un hallazgo asociado con coledocolitiasis con una sensibilidad del $80 \% .{ }^{19}$ Otros hallazgos ecográficos indicativos de obstrucción biliar son la microlitiasis con cálculos vesiculares menores de $5 \mathrm{~mm}$ que tiene mayor riesgo de migración a través del conducto en contraposición con cálculos grandes o único, y la presencia de un cálculo en la vía biliar. ${ }^{20}$ Opciones imagenológicas mejores son la colangiorresonancia o la ecoendoscopia que tienen sensibilidad y especificidad superiores al $90 \% .{ }^{21}$ Son procedimientos menos invasivos pero con mayores costos.

Los hallazgos descritos se han asociado con un aumento de la probabilidad de coledocolitiasis, pero de manera individual cursan con baja sensibilidad ${ }^{22}$, por lo que múltiples estudios han tratado de evaluar el rendimiento de los diferentes predictores de coledocolitiasis. Unos aseguran que hallazgos clínicos como ictericia, coluria y acolia tienen todavía valor para la realización del diagnóstico, tal como se demuestra en el estudio de Ali Yaghoobi y col. ${ }^{23}$ (Tabla 1).

Otros estudios demuestran que los factores bioquímicos y radiológicos siguen jugando un papel principal en la evaluación de los pacientes con sospecha de coledocolitiasis, de tal manera que los más útiles han sido gama glutil transferasa (GGT), fosfatasa alcalina y ALT con una sensibilidad de $84 \%, 79 \%$ y $71 \%$. Aquellos con mayor especificidad son la dilatación de la vía biliar y la amilasa ${ }^{24,25}$ (Tabla 2).

La combinación de los diferentes factores para aumentar el rendimiento de las pruebas también ha sido analizado, llegando a demostrar que en mayores de 55 años con bilirrubina directa superior a $1.9 \mathrm{mg} / \mathrm{dl}$ 


\begin{tabular}{|l|c|c|c|}
\hline \multicolumn{4}{|c|}{ Tabla I. Hallazgos clínicos en colelitiasis y coledocolitiasis (\%) } \\
\hline & Grupo I (Colelitiasis) & Grupo 2 (Coledocolitiasis) & Valor de p \\
\hline Síntomas & 17.2 & & \\
Fiebre & 46.7 & 23.3 & 0.41 \\
Náusea & 31.6 & 56.9 & 0.27 \\
Anorexia & 6.8 & 56.8 & 0.01 \\
Ictericia & 78.3 & 50.8 & $<0.00001$ \\
Dolor en hipocondrio derecho & 2.6 & 50.8 & 0.08 \\
Prurito & & 19.6 & 0.02 \\
Coluria & & 28.8 & 0.00004 \\
Signos e historia clínica & 3.4 & 48.3 & $<0.00001$ \\
Ictericia & & 11.4 & 0.39 \\
Pancreatitis & 3.7 & 11.9 & 0.06 \\
Colangitis & & 2.5 & 0.1 \\
Masa palpable & & & \\
\hline
\end{tabular}

Tomado de Ali Yaghoobi Notash, et al. Preoperative clinical and paraclinical predictors of choledocholithiasis. Hepatobiliary Pancreat Dis Int. June 15, 2008;7(3).

\begin{tabular}{|l|c|c|c|c|c|c|c|}
\hline \multicolumn{6}{|c|}{ Tabla 2. Predictores de coledocolitiasis en colecistectomía laparoscópica } \\
\hline Predictor & Sensibilidad & $\begin{array}{c}\text { Especificidad } \\
\text { ratio }\end{array}$ & $\begin{array}{c}\text { Likehoood } \\
\text { predictivo } \\
\text { positivo }\end{array}$ & $\begin{array}{c}\text { Exactitud } \\
\text { predictivo } \\
\text { negativo }\end{array}$ & Valor & Valor & Valor $p$ \\
\hline CPRE n=199 & 96.0 & 99.1 & 107.3 & 98.0 & 98.8 & 97.4 & 0.000 \\
Ecografían=926 & 35.7 & 97.9 & 8.1 & 93.2 & 58.1 & 94.9 & 0.000 \\
TAC $n=232$ & 74.5 & 89.5 & 7.1 & 86.2 & 66.7 & 92.6 & 0.000 \\
RMN n=32 & 75.0 & 87.5 & 6.0 & 84.4 & 66.7 & 91.3 & 0.002 \\
Diámetro colédoco n=994 & 54.5 & 91.8 & 6.6 & 88.7 & 38.8 & 95.4 & 0.000 \\
Amilasa n=304 & 45.5 & 91.8 & 1.6 & 67.4 & 21.1 & 88.5 & 0.035 \\
GGT n=1002 & 84.1 & 72.0 & 3.0 & 75.7 & 22.4 & 97.9 & 0.000 \\
FA n=1002 & 79.5 & 72.9 & 2.9 & 73.5 & 22.0 & 97.4 & 0.000 \\
BT $n=1002$ & 48.9 & 87.5 & 3.9 & 84.1 & 27.4 & 94.7 & 0.000 \\
ALT $n=1002$ & 71.6 & 68.2 & 2.3 & 68.5 & 17.8 & 96.1 & 0.000 \\
AST n=1002 & 63.6 & 78.7 & 3.0 & 72.4 & 22.3 & 95.7 & 0.000 \\
GGT+FA+BT+ALT+AST $n=1002$ & 87.5 & 53.3 & 1.8 & 63.4 & 15.3 & 97.8 & 0.000 \\
\hline
\end{tabular}

CPRE: colangiopancreatografía retrógrada endoscópica; TAC: tomografía axial computarizada; RMN: resonancia nuclear magnética; GGT: gama glutamil transferasa; FA: fosfatasa alcalina; BT: bilirrubinas totales; ALT: alanina amino transferasa; AST: aspartato amino transferasa; GGT+FA+BT+ALT+AST: al menos una elevación anormal de alguno de los 5 parámetros bioquímicos. Tomado de Ming-Hsun Yang et al. Biochemical predictors for absence of common bile duct stones in patients undergoing laparoscopic cholecystectomy. Surg Endosc. 2008;22:1620-4.

y la dilatación de la vía biliar por ecografía mayor de $7 \mathrm{~mm}$, la probabilidad de coledocolitiasis es de 19 a $38 \%$ con un solo criterio y llega a ser de $72 \%$ cuando se cumplen los $\operatorname{tres}^{26}$ (Figura 1).

Por tal motivo se han creado modelos probabilísticos donde al evaluar los parámetros clínicos, bioquímicos y radiológicos que incluyen la presencia de colangitis, dilatación de la vía biliar con cálculo en el ultrasonido, elevación de la aspartato amino transferasa (AST) y de la bilirrubina directa, se puede tener una probabilidad del $99 \%$ cuando se cumplen todos los criterios y del $7 \%$ cuando ninguno está presente $^{27}$ (Tabla 3).

Dentro de los estudios con mayor poder estadístico encontramos los metanálisis, que muestran la hiperbilirrubinemia como uno de los factores con mayor 


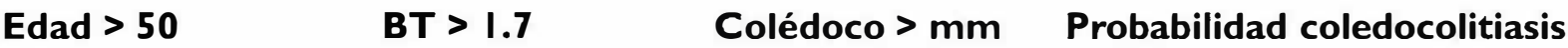

$(+)$

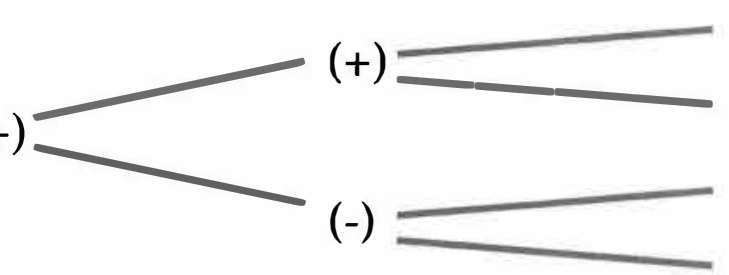

$(-)$

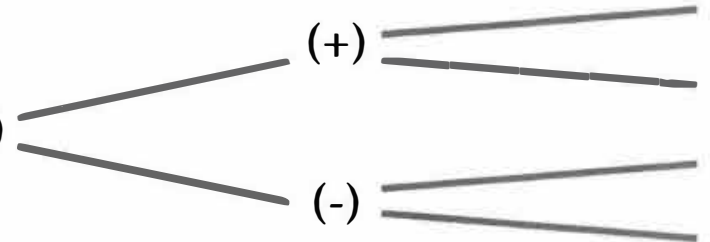

$(+)$

$(-)$

$(+)$

$(-)$

$(+)$

$(-)$

$(+)$

$(-)$
$72 \%$

$50 \%$

$61 \%$

$38 \%$

$49 \%$

$28 \%$

$38 \%$

$19 \%$

Figura I. Predictores de coledocolitiasis en pacientes que serán llevados a colecistectomía. Tomado de Barkun AN, Barkun JS, Fried GM, et al. Useful predictors of bile duct stones in patients undergoing laparoscopic cholecystectomy. McGill Gallstone Treatment Group.Ann Surg 1994;220(1):32-9.

Tabla 3. Probabilidad de coledocolitiasis basada en un patrón de predictores

\begin{tabular}{|c|c|c|c|c|c|}
\hline Modelo & Colangitis & $\begin{array}{l}\text { Dilatación de la } \\
\text { via biliar con } \\
\text { cálculo en ecografía }\end{array}$ & Elevación AST & $\begin{array}{l}\text { Elevación } \\
\text { bilirrubina } \\
\text { conjugada }\end{array}$ & Probabilidad (\%) \\
\hline $\begin{array}{l}1 \\
2 \\
3 \\
4 \\
5 \\
6 \\
7 \\
8 \\
9 \\
10 \\
11 \\
12 \\
13 \\
14 \\
15 \\
16\end{array}$ & $\begin{array}{c}\text { No } \\
\text { Si } \\
\text { No } \\
\text { No } \\
\text { No } \\
\text { Si } \\
\text { Si } \\
\text { Si } \\
\text { No } \\
\text { No } \\
\text { No } \\
\text { Si } \\
\text { Sí } \\
\text { Sí } \\
\text { No } \\
\text { Si }\end{array}$ & $\begin{array}{c}\text { No } \\
\mathrm{Si} \\
\mathrm{Si} \\
\mathrm{No} \\
\mathrm{No} \\
\mathrm{Si} \\
\mathrm{No} \\
\mathrm{No} \\
\mathrm{Si} \\
\mathrm{Si} \\
\mathrm{No} \\
\mathrm{Si} \\
\mathrm{Si} \\
\mathrm{No} \\
\mathrm{Si} \\
\mathrm{Si}\end{array}$ & $\begin{array}{c}\text { No } \\
\text { No } \\
\text { No } \\
\text { Si } \\
\text { No } \\
\text { No } \\
\text { Si } \\
\text { No } \\
\text { Si } \\
\text { No } \\
\text { Si } \\
\text { Si } \\
\text { No } \\
\text { Si } \\
\text { Si } \\
\text { Si }\end{array}$ & $\begin{array}{c}\text { No } \\
\text { No } \\
\text { No } \\
\text { No } \\
\text { Sí } \\
\text { No } \\
\text { No } \\
\text { Sí } \\
\text { No } \\
\text { Si } \\
\text { Si } \\
\text { No } \\
\text { Sí } \\
\text { Sí } \\
\text { Sí } \\
\text { Si }\end{array}$ & $\begin{array}{c}7 \\
43 \\
35 \\
18 \\
28 \\
85 \\
69 \\
80 \\
61 \\
74 \\
53 \\
94 \\
97 \\
92 \\
89 \\
99\end{array}$ \\
\hline
\end{tabular}

Tomado de A.Alponat, C. K. Kum, A. Rajnakova, B. C. Koh, P. M.Y. Goh. Predictive factors for synchronous common bile duct stones in patients with cholelithiasis. Surg Endosc. 1997;11:928-32.

sensibilidad y el hallazgo de cálculo en ultrasonografía y la colangitis como los más específicos. Los valores de laboratorio se ven relegados a unas especificidades, sensibilidades y LR bajos ${ }^{28,29}$ (Tablas 4 y 5 ).
Con todo lo anterior se han desarrollado algoritmos de manejo que buscan orientar el tratamiento y determinar los estudios confirmatorios que disminuyan los riesgos de los pacientes con procedimientos invasivos 


\section{Tabla 4. Sensibilidad y especificidad de los predictores de coledocolitiasis}

\begin{tabular}{|l|c|c|c|c|}
\hline Indicador & Sensibilidad & IC 95\% & Especificidad & IC 95\% \\
\hline Cálculo en el colédoco por ecografía & 0.38 & $0.27-0.49$ & 1.00 & $0.99-1.00$ \\
Colangitis & 0.11 & $0.02-0.19$ & 0.99 & $0.99-1.00$ \\
Ictericia preoperatoria & 0.36 & $0.26-0.45$ & 0.97 & $0.95-0.99$ \\
Colédoco dilatado por ecografía & 0.42 & $0.28-0.56$ & 0.96 & $0.94-0.98$ \\
Amilasa & 0.11 & $0.02-0.20$ & 0.95 & $0.93-0.98$ \\
Pancreatitis & 0.10 & $0.08-0.12$ & 0.95 & $0.93-0.97$ \\
Ictericia & 0.39 & $0.29-0.49$ & 0.92 & $0.88-0.97$ \\
Bilirrubinas & 0.69 & $0.48-0.90$ & 0.88 & $0.84-0.92$ \\
Fosfatasa alcalina & 0.57 & $0.46-0.69$ & 0.86 & $0.78-0.94$ \\
Colecistitis & 0.50 & $0.11-0.89$ & 0.76 & $0.45-1.00$
\end{tabular}

Tomado de Abboud P, Malet P, Berlin J.Predictors of common bile duct stones prior to cholecystectomy a meta-analysis. Gastrointestinal Endoscopy - Volume 44, Issue 4 (October 1996).

Tabla 5. Razones de probabilidad (Likelihood ratios)

\begin{tabular}{|l|c|c|c|c|}
\hline Indicador & LR+ & $95 \% \mathbf{C l}$ & LR- & $\mathbf{9 5 \%} \mathbf{C l}$ \\
\hline Cálculo en el colédoco por ecografía & 18.3 & $9.0-37.1$ & 0.93 & $0.89-0.96$ \\
Colangitis & 13.6 & $7.5-24.8$ & 0.70 & $0.63-0.77$ \\
Ictericia preoperatoria & 10.1 & $7.3-13.9$ & 0.69 & $0.64-0.74$ \\
Colédoco dilatado por ecografía & 6.9 & $5.6-8.6$ & 0.77 & $0.72-0.82$ \\
Amilasa & 4.8 & $4.4-5.3$ & 0.54 & $0.49-0.60$ \\
Pancreatitis & 3.9 & $3.3-4.5$ & 0.82 & $0.78-0.86$ \\
Ictericia & 2.6 & $2.4-2.9$ & 0.65 & $0.59-0.71$ \\
Bilirrubinas & 2.1 & $1.6-2.7$ & 0.96 & $0.94-0.98$ \\
Fosfatasa alcalina & 1.6 & $1.4-1.9$ & 0.94 & $0.87-1.01$ \\
Colecistitis & 1.5 & $1.1-2.1$ & 0.99 & $0.96-1.01$ \\
\hline
\end{tabular}

Tomado de Abboud P, Malet P, Berlin J.Predictors of common bile duct stones prior to cholecystectomy a meta-analysis. Gastrointestinal Endoscopy - Volume 44, Issue 4 (October 1996).

y reduzcan los costos con pruebas no invasivas realizadas de manera injustificada. ${ }^{30} \mathrm{El}$ algoritmo que se propone estratifica el riesgo de presentar coledocolitiasis en pacientes con colelitiasis que serán llevados a colecistectomía acorde con el comportamiento de los predictores clínicos, radiológicos y bioquímicos descritos, ${ }^{31}$ de tal manera que los que cursan con bajo riesgo tienen una probabilidad menor del $10 \%$ de presentar cálculos en el colédoco, por lo que no se recomiendan evaluaciones adicionales que aumenten los riesgos y costos. En el riesgo intermedio la probabilidad de coledocolitiasis es del 10 al 50\%, por lo tanto, se recomiendan evaluaciones adicionales que permitan definir la necesidad de procedimientos inva- sivos, y en el riesgo alto la coledocolitiasis es factible en más del $50 \%$ por lo que se requieren procedimientos que además de la confirmación diagnóstica permitan su manejo ${ }^{31}$ (Figura 2). Los predictores evaluados en este algoritmo se clasificaron como muy fuertes ante la presencia de cálculo en el colédoco en la ecografía hepatobiliar, bilirrubina total mayor de $4 \mathrm{mg} / \mathrm{dl}$ y la presencia de colangitis. Fuertes cuando la vía biliar dilatada con colédoco mayor de $7 \mathrm{~mm}$ y bilirrubinas totales de 1.8 a $4 \mathrm{mg} / \mathrm{dl}$; moderados se consideraron la alteración de otro marcador de la función hepática, edad superior a 55 años y que presenten pancreatitis aguda de origen biliar. Se considera entonces de alto riesgo a quienes presentan alguno de los predictores 
muy fuertes o ambos de los considerados fuertes. De bajo riesgo a los que no presentan ningún predictor y el resto de pacientes son de riesgo intermedio. ${ }^{31}$

\section{Manejo}

Los cálculos del colédoco pueden migrar desde la vesícula o formarse en forma primaria dentro de la propia vía biliar y constituyen un riesgo ya que pueden provocar cólicos biliares, ictericia obstructiva, colangitis o pancreatitis aguda. Del 10 al $15 \%$ de los que sufren colelitiasis presentan coledocolitiasis en algún momento del tratamiento ${ }^{32}$, y del 18 al $33 \%$ de los que cursan con colangitis o pancreatitis aguda. ${ }^{33}$ De estos pacientes en cerca de un tercio los cálculos obstructivos pueden eliminarse en dos meses sin ninguna intervención. ${ }^{34} \mathrm{El}$ resto precisan endoscopia o cirugía para aliviar la obstrucción. ${ }^{35}$
La CPRE (colongiopancreatografía retrógrada endoscópica) nació en la década de los setenta como técnica alterna para la extracción de cálculos, pero no tuvo mucha acogida porque como en ese tiempo todas las colecistectomías se realizaban abiertas, se podía de una vez ejecutar la exploración de las vías biliares. ${ }^{36}$ Con el advenimiento de la colecistectomía laparoscópica la CPRE volvió a tener cabida. Las indicaciones absolutas de CPRE preoperatoria son colangitis tóxica y pancreatitis biliar severa. ${ }^{37}$

El 85 a 90\% de los cálculos en la vía biliar se pueden extraer mediante canastilla. ${ }^{38}$ Las complicaciones del procedimiento son pancreatitis $5,4 \%$, sangrado $2 \%$, colangitis $1 \%$, colecistitis $0,5 \%$ y perforación duodenal en $0,3 \% .{ }^{39}$ Estudios en pacientes que solo se sometieron a CPRE muestran que el 75 a $85 \%$ permanecen sin síntomas en un seguimiento a 70 meses. ${ }^{40}$ Solo el 10 a $15 \%$ de las CPRE son fallidas. ${ }^{41}$ En estas la

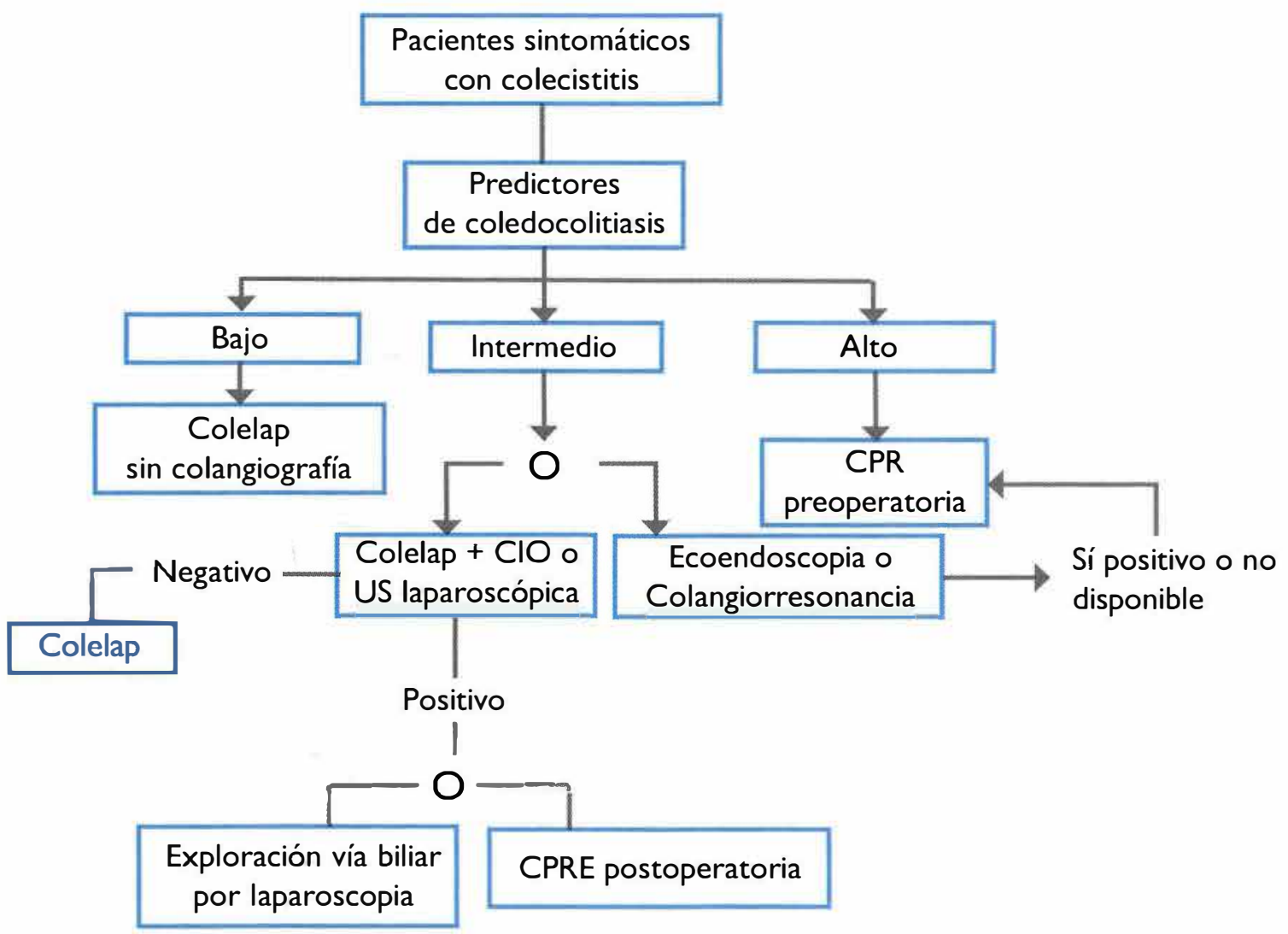

Figura 2. Algoritmo para el manejo de pacientes con sospecha de coledocolitiasis. Tomado de Byrne MF, McLoughlin MT, Mitchell RM, et al. Surg Endosc 2009;23:1933-7. 
vía quirúrgica es la opción, la cual ha sido la técnica tradicional para la extracción de cálculos.

Durante colecistectomía laparoscópica se pueden extraer los cálculos por varias vías, la transcística con catéter de Fogarty por lo regular remueve cálculos de menos de $8 \mathrm{~mm} .{ }^{42}$ Esta técnica es exitosa en un $80-90 \%$ de las personas; de igual forma el lavado con sonda del conducto puede evacuar pequeños cálculos en la ampolla. ${ }^{43}$ Se puede introducir un coledocoscopio por el cístico y extraer los cálculos por el puerto de trabajo de este último. ${ }^{44}$

En pacientes con múltiples cálculos o que no es posible extraerlos por el cístico, se puede realizar coledocotomía y exploración vía laparoscópica con porcentaje de éxito de $85-95 \% .{ }^{45} \mathrm{La}$ indicación de tubo en $\mathrm{T}$ ya no es tan clara, tanto que algunos autores no lo colocan sin complicaciones aparentes. ${ }^{46}$

Otra opción de tratamiento es dejar una sonda transcística y realizar luego de la colecistectomía laparoscópica una CPRE. Son buenos los resultados reportados pero se corre el riesgo de tener que someter al paciente a una segunda reintervención. ${ }^{47}$ Donde no hay posibilidad de CPRE o esta es fallida y no se cuenta con la opción laparoscópica, o durante alguna complicación de estos procedimientos, siempre está la opción de realizar una cirugía abierta con exploración formal de las vías biliares, para lo cual hay varias opciones. Una es la coledocotomía con extracción e instrumentación de la vía biliar, indicada en coledocolitiasis con colédoco menor de $2 \mathrm{~cm} .{ }^{48}$ Otra opción es una esfinteroplastia pero tiene indicaciones muy claras: cálculo impactado en la ampolla o coledocolitiasis múltiple. ${ }^{49}$ En los pacientes con colédoco de más de $2 \mathrm{~cm}$ se recomienda la derivación de la vía biliar ya sea con hepaticoyeyunostomía o coledocoduodenostomía. ${ }^{50}$

\section{Conclusión}

El enfoque de los pacientes con sospecha de coledocolitiasis requiere un cuidadoso análisis y consideración de los diferentes elementos en cuestión, ya que aunque el porcentaje de presentación es más bien bajo, sus complicaciones como los síntomas recurrentes, pan- creatitis y colangitis no pueden menospreciarse, todo esto dentro de un contexto donde se deben tener en cuenta los costos y complicaciones de la evaluación complementaria de la vía biliar, la cual debe reducirse a lo mínimo en un sistema de salud como el nuestro donde la utilización de recursos es limitada y regida por el rigor científico.

\section{Referencias}

1. Elwood DR. Cholecystitis. Surg Clin North Am. 2008 Dec;88(6):1241-52, viii.

2. Glasgow RE, Cho M, Hutter MM, Mulvihill SJ. The spectrum and cost of com plicated gallstone disease in California. Arch Surg. 2000 Sep;135(9):1021-5.

3. Cervantes J, Rojas G, Anton J. Conductas actuales ante la litiasis coledociana. Cirugía y cirujanos. 1999;67,49-54. Ref Type: Magazine Article

4. Morgenstern L. A history of choledochotomy. Bile ducts and bile duct stones. 1997.

5. Schwarz S. Principios de cirugía. 4 ed. 1997.

6. Blumgart H. Surgical and radiology anatomy of the liver, biliary tract and pancreas. 4 ed. 2006

7. Skandalakis J. Anatomía y técnica quirúrgica. 2002.

8. Stapleton GN, Hickman R, Terblanche J. Blood supply of the right and left hepatic ducts. Br J Surg. 1998 Feb;85(2):202-7.

9. Saint JH. The epicholedochal venous plexus and its importance as a means of identifying the common duct during operations on the extrahepatic biliary tract. Br J Surg. 1961 Mar;48:489-98.

10. Vellar ID. Preliminary study of the anatomy of the venous drainage of the intrahepatic and extrahepatic bile ducts and its relevance to the practice of hepatobiliary surgery. ANZ J Surg. 2001 Jul;71(7):418-22.

11. Hand BH. Anatomy and function of the extrahepatic biliary system. Clin Gastroenterol. 1973 Jan;2(1):3-29.

12. Tazuma S. Gallstone disease: Epidemiology, pathogenesis, and classification of biliary stones (common bile duct and intrahepatic). Best Pract Res Clin Gastroenterol. 2006;20(6): 1075-83.

13. Lambou-Gianoukos S, Heller SJ. Lithogenesis and bile metabolism. Surg Clin North Am. 2008 Dec;88(6):1175-94, vii

14. Collins C, Maguire D. Ireland A, Fitzgerald E, O'Sullivan GC. A prospective study of common bile duct calculi in patients undergoing laparoscopic cholecystectomy: natural history of choledocholithiasis revisited. Ann Surg. 2004 Jan;239(1):28-33.

15. Yang MH, Chen TH, Wang SE, Tsai YF, Su CH, Wu CW, et al. Biochemical predictors for absence of common bile duct stones in patients undergoing laparoscopic cholecystectomy. Surg Endosc. 2008 Jul;22(7):1620-4.

16. Peng WK, Sheikh Z, Paterson-Brown S, Nixon SJ. Role of liver function tests in predicting common bile duct stones in acute calculous cholecystitis. Br J Surg. 2005 Oct:92(10):1241-7.

17. Onken JE, Brazer SR, Eisen GM, Williams DM, Bouras EP, DeLong ER, et al. Predicting the presence of choledocholithiasis in patients with symptomatic cholelithiasis. Am J Gastroenterol. 1996 Apr;91(4):762-7.

18. Lapis JL. Orlando RC, Mittelstaedt CA, Staab EV. Ultrasonography in the diag nosis of obstructive jaundice. Ann Intern Med. 1978 Jul;89(1):61-3.

19. Costi R. Sarli L, Caruso G, Iusco D, Gobbi S, Violi V, et al. Preoperative ultrasonographic assessment of the number and size of gallbladder stones: is it a useful predictor of asymptomatic choledochal lithiasis? J Ultrasound Med. 2002 Sep;21(9):971-6. 
20. Demartines N, Eisner L, Schnabel K, Fried R, Zuber M, Harder F. Evaluation of magnetic resonance cholangiography in the management of bile duct stones. Arch Surg. 2000 Feb;135(2): 148-52.

21. Mercer S, Singh S, Paterson 1. Selective MRCP in the management of suspected common bile duct stones. HPB (Oxford). 2007;9(2):125-30.

22. Bose SM, Mazumdar A, Prakash VS, Kocher R, Katariya S, Pathak CM. Evaluation of the predictors of choledocholithiasis: comparative analysis of clinical, biochemical, radiological, radionuclear, and intraoperative parameters. Surg Today. $2001 ; 31(2): 117-22$.

23. Notash AY, Salimi J, Golfam F, Habibi G, Alizadeh K. Preoperative clinical and paraclinical predictors of choledocholithiasis. Hepatobiliary Pancreat Dis Int. 2008 Jun;7(3):304-7.

24. Yang $\mathrm{MH}$, Chen TH, Wang SE, Tsai YF, Su CH, Wu CW, et al. Biochemical predictors for absence of common bile duct stones in patients undergoing laparoscopic cholecystectomy. Surg Endosc. 2008 Jul;22(7): 1620-4.

25. Shiozawa S, Tsuchiya A, Kim DH, Usui T, Masuda T, Kubota K, et al. Useful predictive factors of common bile duct stones prior to laparoscopic cholecystectomy for gallstones. Hepatogastroenterology. 2005 Nov:52(66):1662-5.

26. Barkun AN, Barkun JS, Fried GM, Ghitulescu G, Steinmetz O, Pham C, et al. Useful predictors of bile duct stones in patients undergoing laparoscopic cholecystectomy. McGill Gallstone Treatment Group. Ann Surg. 1994 Jul;220(1):329.

27. Alponat A, Kum CK, Rajnakova A, Koh BC, Goh PM. Predictive factors for synchronous common bile duct stones in patients with cholelithiasis. Surg Endosc. 1997 Sep;11 (9):928-32.

28. Abboud PA, Malet PF, Berlin JA, Staroscik R, Cabana MD, Clarke JR, et al. Predictors of common bile duct stones prior to cholecystectomy: a meta-analysis. Gastrointest Endosc. 1996 Oct;44(4):450-5.

29. Tse F, Barkun JS, Barkun AN. The elective evaluation of patients with suspected choledocholithiasis undergoing laparoscopic cholecystectomy. Gastrointest Endosc. 2004 Sep;60(3):437-48.

30. Eisen GM, Dominitz JA, Faigel DO, Goldstein JL, Kalloo AN, Petersen BT, et al. An annotated algorithm for the evaluation of choledocholithiasis. Gastrointest Endosc. 2001 Jun;53(7):864-6.

31. Byme MF, McLoughlin MT, Mitchell RM, Gerke H, Kim K, Pappas TN, et al. For patients with predicted low risk for choledocholithiasis undergoing laparoscopic cholecystectomy, selective intraoperative cholangiography and postoperative endoscopic retrograde cholangiopancreatography is an effective strategy to limit unnecessary procedures. Surg Endosc. 2009 Sep;23(9): 1933-7.

32. Ko CW, Lee SP. Epidemiology and natural history of common bile duct stones and prediction of disease. Gastrointest Endosc. 2002 Dec;56(6 Suppl):S165S169.

33. Cohen ME, Slezak L, Wells CK, Andersen DK, Topazian M. Prediction of bile duct stones and complications in gallstone pancreatitis using early laboratory trends. Am J Gastroenterol. 2001 Dec;96(12):3305-11
34. Collins C, Maguire D, Ireland A, Fitzgerald E, O'Sullivan GC. A prospective study of common bile duct calculi in patients undergoing laparoscopic cholecystectomy: natural history of choledocholithiasis revisited. Ann Surg. 2004 Jan;239(1):28-33.

35. Verbesey JE, Birkett DH. Common bile duct exploration for choledocholithiasis. Surg Clin Nortb Am. 2008 Dec;88(6): 1315-28, ix.

36. Joyce AM, Heiss FW. Endoscopic evaluation and therapies of biliary disorders. Surg Clin North Am. 2008 Dec;88(6):1221-40, viii.

37. NIH state-of-the-science statement on endoscopic retrograde cholangiopancreatography (ERCP) for diagnosis and therapy. NIH Consens State Sci Statements. 2002 Jan 14;19(1):1-26.

38. Hintze RE, Adler A, Veltzke W. Outcome of mechanical lithotripsy of bile duct stones in an unselected series of 704 patients. Hepatogastroenterology. 1996 May;43(9):473-6.

39. Freeman ML, DiSario JA, Nelson DB, Fennerty MB, Lee JG, Bjorkman DJ, et al. Risk factors for post-ERCP pancreatitis: a prospective, multicenter study. Gastrointest Endosc. 2001 Oct;54(4):425-34.

40. Masci E, Toti G, Mariani A, Curioni S, Lomazzi A, Dinelli M, et al. Complications of diagnostic and therapeutic ERCP: a prospective multicenter study. Am J Gastroenterol. 2001 Feb;96(2):417-23.

41. Petelin JB. Laparoscopic common bile duct exploration. Surg Endosc. 2003 Nov;17(11):1705-15.

42. Hampson LG, Fried GM, Stets J, Ayeni OR, Bourdon-Conochie F. Common bile duct exploration: indications and results. Can J Surg. 1981 Sep;24(5):455-7.

43. Martin DJ, Vernon DR, Toouli J. Surgical versus endoscopic treatment of bile duct stones. Cochrane Database Syst Rev. 2006 Apr;\% 19;(2):CD003327.

44. Petelin JB. Techniques and Cost of Common Bile Duct Exploration. Semin Laparosc Surg. 1997 Mar;4(1):23-33.

45. Tai CK, Tang CN, Ha JP, Chau CH, Siu WT, Li MK. Laparoscopic exploration of common bile duct in difficult choledocholithiasis. Surg Endosc. 2004 Jun; 18(6):910-4.

46. Gurusamy KS, Samraj K. Primary closure versus T-tube drainage after laparoscopic common bile duct stone exploration. Cochrane Database Syst Rev. 2007 Jan 24;(1):CD005641.

47. Garg PK, Tandon RK, Ahuja V, Makharia GK, Batra Y. Predictors of unsuccessful mechanical lithotripsy and endoscopic clearance of large bile duct stones. Gastrointest Endosc. 2004 May;59(6):601-5.

48. Zinner M, Ashley S. Maingont: operaciones abdominales. 2008.

49. Suter M, Jayet C, Richard A, Gillet M. [Current status of surgical transduodenal papillotomy]. Helv Chir Acta 1994 Apr:60(4):671-8.

50. Rivera JA, Rattner DW, Fernandez-del CC, Warshaw AL. Surgical approaches to benign and malignant tumors of the ampulla of vater. Surg Oncol Clin N Am. $1996 \mathrm{Jul} ; 5(3): 689-711$. 